\title{
Ein Fisch auf dem Trockenen?
}

\author{
Wasserkraftwerke sind ökologisch umstritten. Sie verfevern keine fossilen En- \\ ergieträger, versperren aber Fischen den Weg bei der Wanderschaft. Die \\ Lösung des Konflikts liegt in der fischgerechten Gestaltung der Kraftwerke. \\ Die dazu notwendigen rechtlichen Mittel sind durch das Erneverbare Energien \\ Gesetz und die Wasserrahmenrichtlinie gegeben.
}

$\mathrm{D}$ Von Bettina Keite ie Wasserkraft hat einen guten Ruf. Sie ist eine erneuerbare Energie, verursacht keine Treibhausgase und trägt damit zum Klimaschutz bei. Die technisch ausgereifte Wasserkraft erhitzt aber trotz ihres Klimaschutzeffekts die Gemüter der Naturschützer und steht im Spannungsfeld der Interessen von Klimaschutz, Energiegewinnung und Naturschutz.

Grundsätzlich ist der Ausbau erneuerbarer Energien sinnvoll, da es aus Gründen des Klimaschutzes und der Gefahren der Atomenergie keine Alternative zu ihnen gibt. Bei der Wasserkraft sind aber enge ökologische Grenzen gesetzt, da die Gewässerökologie durch Wasserkraftnutzung stark beeinflusst wird. Daher müssen nach Auffassung des Naturschutzbundes (NABU) naturnahe Flussabschnitte als Tabuzonen für die Wasserkraftnutzung gelten.

Die Chancen der Wasserkraft liegen in der Modernisierung bestehender Anlagen, die aber immer mit einer Verbesserung der ökologischen Situation und der Erreichung der Durchgängigkeit fuir wandernde Lebewesen verbunden sein muß.

\section{- Kritikpunkt Durchgängigkeit}

Eine Laufwasserkraftanlage hat eine Barrierewirkung im Fließgewässer, da ein Wehr zur Herstellung und Regulierung des Wasserstaus genutzt wird. Die Stromerzeugung findet durch eine von Turbinen getriebene Wasserkraftanlage statt, die am Wehr baulich integriert ist. Bei Ausleitungskraftwerken, einer weit verbreiteten Typvariante von Wasserkraftwerken, wird das Wasser ebenfalls durch ein Wehr aufgestaut, dann jedoch durch einen zusätzlich geschaffenen Triebwasserkanal Richtung Maschinenhaus zur Stromproduktion umgeleitet. Aufgrund der Wasserumleitung fließt dann im ursprünglichen Flussbett nur noch eine Restwassermenge.
Die Kritikpunkte an der Wasserkraft liegen daher aus Naturschutzsicht hauptsächlich in der gestörten Durchgängigkeit der Fließgewässer und in den Folgen für die aquatischen Lebensgemeinschaften. Bei Wasserkraftanlagen mit Ausleitungsstrecken kommt zusätzlich noch die Problematik des geringen Wasserabflusses in der Restwasserstrecke hinzu. Andererseits deckt Wasserkraft in klimafreundlicher Weise 4,7 Prozent unseres Strombedarfs, da bei der Energieerzeugung kein Kohlendioxid freigesetzt wird.

In diesem umweltpolitischen Spannungsfeld haben sich über Jahrzehnte Konflikte manifestiert. Das am 01.08.2004 in Kraft getretene Gesetz zur Neuregelung des Rechts der Erneuerbaren Energien im Strombereich (EEG) und die Umsetzung der Europäischen Wasserrahmenrichtlinie (WRRL) geben aber die rechtlichen Voraussetzungen zur Überwindung dieser Konfliktsituation.

\section{- Folgen für den Fluss}

Die Leistung von Wasserkraftanlagen hängt in erster Linie von der vorhandenen Wassermenge und der nutzbaren Fallhöhe ab, die über ein Querbauwerk mit dem Zweck des Gewässeraufstaus erzielt wird. Angepasst an die jeweilige Wassermenge und Fallhöhe werden unterschiedliche Typen von Wasserkraftanlagen und Turbinen entwickelt.

Die Barrierewirkung von Laufwasserkraftanlagen greift tief in das ökologische System von Flüssen und Bächen ein. Damit gerät die Wasserkraft auch in einen Zielkonflikt mit der Europäischen Wasserrahmenrichtlinie und ihrer Vorgabe, einen guten ökologischen Zustand an allen Oberflächengewässern der EU bis zum Jahr 2015 zu erreichen. Die WRRL fordert die Wiederherstellung der Durchgängigkeit für alle Oberflächengewässer. In Bezug auf die Wasserkraftanlagen umfasst die fehlende Durchgängigkeit erstens die Unterbrechung der Durchwanderbarkeit von Fließgewässers durch
Querbauwerke und zweitens die Folgen dieser Barrierewirkung auf die Strömungs- und Sedimentverhältnisse des Fließgewässers.

Die Durchwanderbarkeit beinhaltet, dass sich Fische und andere Wasserorganismen wieder über längere Strecken im Gewässer bewegen können; das gilt nicht nur für den Aal oder Lachs als prominente Vertreter der Wanderfischfraktion. Die beste Wanderhilfe ist aus Naturschutzsicht natuirlich die, die gar nicht benötigt wird, da kein Wehr vorhanden ist. Eine Anlage zur Herstellung der Durchwanderbarkeit kann daher immer nur eine Notlösung darstellen. Die zweitbeste Option ist die naturnahe Wanderhilfe, da technische Anlagen wie Beckenpässe keinen Beitrag zur naturnahen Gewässerstrukturierung leisten. Bei Fischaufstiegen zählen dazu Umgehungsgerinne oder raue Rampen. Da Fische nicht nur ein Gewässer hinaufwandern, sondern auch hinab, müssen an den Querbauten Möglichkeiten zum Fischabstieg geschaffen werden. Aufgrund der unterschiedlichen hydraulischen Voraussetzungen können Fische nicht den Fischaufstieg auch zum Fischabstieg nutzen. Naturverträgliche Fischabstiege befinden sich noch in der Entwicklungsphase. Verstärkt setzt sich die Erkenntnis durch, dass der klassische „Fischabstieg“ durch die Turbine hohe Mortalitätsraten bei Fischen mit sich bringt.

Die Barrierewirkung der Wehranlagen wirkt sich auf die Strömungs- und Geschiebeverhältnisse des Fließgewässers aus. Der Gewässerstau wirkt daher als Sedimentfalle, da die Schwebteilchen aufgrund der reduzierten Fließgeschwindigkeit absinken können. Zudem verändern sich im Staubereich die biochemischen und physikalischen Gewässereigenschaften wie Temperatur und Sauerstoffgehalt. Die Unterbrechung des Geschiebetransports zieht eine vermehrte Erosion unterhalb der Stauanlage nach sich. Zudem ist der natürliche Wechsel zwischen Niedrig- und Hochwasserphasen abgepuffert. Insgesamt hat die Barrierewirkung der Anlagen eine vollkommen anders geprägte Abflusscharakteristik zur Folge.

Bei Ausleitungskraftwerken existiert ein zusätzlicher Kritikpunkt, da aufgrund der Wasserumleitung in den Triebwasserkanal im ursprünglichen Flusslauf eine so genannte Mindestwasserregelung erforderlich wird. Aus Naturschutzsicht muss daher der Mindestwasserabfluss habitatbezogen für die Restwasserstrecke festgelegt werden. Ansonsten besteht die Gefahr, dass sich die Lebewesen im ursprünglichen Flussbett buchstäblich ,auf dem Trockenen“ befinden. 


\section{- Chancen für den Fluss}

Nach Ansicht des NABU steht der energiepolitische Nutzen der kleinen Wasserkraft in keinem Verhältnis zu den damit verbundenen ökologischen Schäden. Daher kann und darf ein Ausbau nur über Modernisierungsmaßnahmen an bestehenden Anlagen vollzogen werden, wenn parallel zur Modernisierung eine ökologische Aufwertung stattfindet. Diese Forderung wird vom neuen EEG aufgegriffen, das als erstes Energiegesetz Bezug auf wasserrechtliche Regelungen nimmt. Dies ist eine historische Chance, die Konflikte um die Wasserkraftnutzung zu überwinden, da das neue EEG die Vergütung des aus Wasserkraft gewonnenen Stroms an ökologische Kriterien koppelt. Ein Schritt, der nicht nur aus ökologischer Sicht sinnvoll ist, sondern als rechtliche Maßgabe der WRRL und der nationalen Wassergesetze Beachtung finden muss.

Bezüglich Laufwasserkraftanlagen bis $500 \mathrm{~kW}$ muss bei der Genehmigung von Neuanlagen $a b$ 2008 ein guter ökologischer Zustand oder eine ökologische Verbesserung gegenüber dem Vorzustand des Gewässers nachgewiesen werden. Ebenfalls ab 2008 gilt für diese Anlagen die Auflage, dass neu in Betrieb zu nehmende Anlagen einen räumlichen Zusammenhang zu einer ganz oder teilweise bestehenden Staustufe beziehungsweise Wehranlage aufweisen müssen. Dies bedeutet, dass ab 2008 keine Wasserkraftanlagen bis zu einer Leistung von $500 \mathrm{~kW}$ an unverbauten Standorten mehr genehmigt werden und an Standorten mit einer bestehenden Querverbauung auch nur dann, wenn der gute ökologische Zustand oder eine ökologische Verbesserung gemäß WRRL attestiert ist. Als Nachweis gilt die Vorlage der behördlichen wasserrechtlichen Zulassung. Allerdings wäre eine Funktionskontrolle der Wanderhilfen zur Sicherstellung der Durchgängigkeit wünschenswert, da die Erfahrung zeigt, dass auch schön angelegte Umgehungsgerinne aus hydraulischen Gründen nicht immer funktionieren. Des Weiteren spielt aus Naturschutzsicht die Naturnähe der Wanderhilfen eine große Rolle. Es bleibt, auf eine strenge Auslegung der WRRL und der in Deutschland geltenden wasserrechtlichen Gesetze zu hoffen.

\section{Fazit}

Auch heute müssen zu genehmigende Wasserkraftanlagen schon den ökologischen Standards des europäischen und deutschen Wasserrechts genügen, so dass mit der Aufnahme der ökologischen Kriterien kein Neuland betreten wird, sondern dies nur mit der Vergütung in Zusammenhang gesetzt wird. Das neue EEG schreibt mit einer Vergütung von 9,67 Eurocent für Anlagen bis $500 \mathrm{~kW}$ eine um zwei Eurocent höhere Vergütung gegenüber dem Vorgänger-EEG vor, wenn die vorgegebenen Standards eingehalten werden. Auf diesem Weg werden finanzielle Anreize zur Modernisierung der Wasserkraftanlagen geschaffen. Aus Sicht des Naturschutzes ist das neue EEG ein wichtiger und richtiger Schritt, denn die Konflikt- lösung zwischen Wasserkraftbetreibern und $\mathrm{Na}$ tur- und Umweltschutz kann nur in der naturintegrierten Energienutzung liegen.

Entsprechend der WRRL Artikel $1 \mathrm{a}$ ist eine weitere Verschlechterung der Gewässer zu vermeiden und deren Zustand zu schützen und zu verbessern. Da der Neubau von kleinen Wasserkraftanlagen umweltbelastender sein kann als die Modernisierung bestehender größerer Kraftwerke, greift in diesen Fällen das Verschlechterungsverbot der WRRL. Damit sind Berechnungen des technisch nutzbaren Wasserkraftpotenzials für Neustandorte irrelevant. Nach Ansicht des NABU besteht die Handlungsoption, durch Modernisierung Potenziale an bestehenden Anlagen verstärkt auszuschöpfen, wenn gleichzeitig eine ökologische Verbesserung erzielt wird. In Deutschland gibt es kaum noch weit gehend natürliche Fließgewässer und diese sollten erhalten bleiben.

\section{Die Autorin}

Bettina Keite ist Wasser-Expertin im Fachbereich Naturschutz und Umweltpolitik beim Naturschutzbund (NABU) Bundesverband.

Kontakt: NABU, Herbert-Rabius-Str. 26,

53225 Bonn. Tel. 0228-4036173,

E-Mail: Bettina.Keite@NABU.de

\section{Die Sonnenseiten der Landschaft}

\section{Solaranlagen brauchen Platz, sonst können sie keine Energie erzeugen. Mit ökologisch verantwortlichen Solarparks können Freiflächen sogar aufgewertet werden.}

F 1 Von Nikolaus Krane ür die erfolgreiche Realisierung eines umweltgerechten Solarparks ist von entscheidender Bedeutung, Anwohner und regionale Umweltinitiativen möglichst frühzeitig und umfassend über das Projekt zu informieren. Transparente Kommunikation und ein konstruktiver Dialog führen zu deutlich höherer Akzeptanz bei den Bürgern. Um Standortentwicklung und spätere Kapitaleinwerbung für Solarfonds erfolgreich durchführen zu können, ist es für den Durchbruch der Photovoltaik notwendig, dass viele Bürger vor Ort hinter dem Projekt stehen. Zum einen ist eine positive Aufnahme der Solaranlage bei den Anrainern fördernd für die allgemeine Akzep$\tan z$ des Solarparks wie auch künftiger Projekte. Bei den 13 bisher von der voltwerk realisierten Solarprojekten hat sich darüber hinaus gezeigt, dass viele Anwohner aus der näheren Umgebung des Solarparks den Solarprojekten sehr aufge- schlossen gegenüberstehen und sich finanziell daran beteiligen. Aus diesem Grund hat die voltwerk gesonderte Konditionen für die Bürger rund um die Solarparks erarbeitet.

Solare Großprojekte sind für die Weiterentwicklung der umwelt-freundlichen Zukunftstechnologie Photovoltaik zwingend notwendig, um den Einstieg in die Massenproduktion zur Kostensenkung der Komponenten voranzutreiben. Von dieser Entwicklung profitieren gerade auch viele Tausend Betreiber privater Solardächer. Dank der ansteigenden Produktionsmengen der Hersteller können sie künftig mit niedrigen Preisen für Solarmodule rechnen. Doch die Suche nach geeigneten Großdächern gestaltet sich recht schwer. Die Solartechnik stellt hohe Anforderungen an große Dachflächen. Einerseits müssen die Dächer statisch geeignet sein. Zudem müssen sie für die nächsten 20 Jahre zur Verfiigung stehen, eine Bindung, auf die sich Dacheigner oft nicht einlassen. 
(c) 20I0 Authors; licensee IÖW and oekom verlag. This is an article distributed under the terms of the Creative Commons Attribution Non-Commercial No Derivates License (http://creativecommons.org/licenses/by-nc-nd/3.o/), which permits unrestricted use, distribution, and reproduction in any medium, provided the original work is properly cited. 